\title{
T-BLAST Architecture for the IEEE 802.11b context
}

\author{
Andrea Bernacchioni, Enrico Del Re, Romano Fantacci, Laura Pierucci \\ University of Florence \\ Department of Electronics and Telecommunications \\ V.S.Marta,3 \\ 50139 Firenze - ITALY \\ E-mail:pierucci@lenst.det.unifi.it
}

\begin{abstract}
Recent research addresses towards multi-transmit, multi-receive antenna scheme to improve the performance in high data rate wireless communications. In this paper, we examine the combination of Bell-Labs Layered Space Time (BLAST) wireless architecture and the iterative demapping and decoding, the Turbo decoding principle. This structure, known as TBLAST, was evaluated according to the IEEE 802.11b standard requirements for Wireless $L A N$. The paper considers the design of the modified 'soft' Complementary Code Keying (CCK) modulation/demodulation scheme more suitable for the iterative interference cancellation receiver and shows the performance evaluation of this T-BLAST and SOFT CCK MODEM in the specific environment of the IEEE 802.11b standard. The paper also presents the throughput versus distance to receiver varying the number of transmit antennas in the indoor environment.
\end{abstract}

\section{INTRODUCTION}

High speed data transmission requires an increase of channel capacity and spectral efficiency. The BLAST techniques can achieve these demands by using multiple transmitting and receiving antennas over wireless channels for a fixed total transmit power. The basic idea is to transmit different signals simultaneously on different antennas and this spatial diversity relies on a rich scattering matrix channel.

The major source of channel impairment in a spatial multiplexing scheme is co-antenna interference (CAI). To mitigate the degrading effects of CAI, a robust multi-transmit multi-receive system using the combination of Turbo decoding principles and V-BLAST was analyzed in literature [1], [2], called TBLAST.

In this paper, we consider the use of the simplified iterative interference receiver as in [2], in the specific context of the IEEE $802.11 \mathrm{~b}$ standard.

The IEEE $802.11 \mathrm{~b}$ standard adopts high data rate with bit rates up to $11 \mathrm{Mbps}$. For achieving data rate greater than $2 \mathrm{Mbps}$, the IEEE $802.11 \mathrm{~b}$ standard specifies the Complementary Code Keying (CCK) modulation scheme.

The paper presents a new method combining a modified 'soft' CCK modulation/demodulation scheme using the iterative information of T-BLAST. Finally, the performance results of T-BLAST structure in the IEEE $802.11 \mathrm{~b}$ environment and the throughput versus distance to receiver varying the number of transmit antennas are shown.

\section{SYSTEM MODEL}

A single data stream is demultiplexed into $M$ substreams, where $\mathrm{M}$ is the number of transmitting antennas, and each substream is then encoded into symbols and fed to its respective transmitter. Transmitters 1-M operate in co-channel way at symbol rate $R_{s}=1 / T_{s}$ symbols/sec, with synchronized symbol timing. It's assumed that the same constellation is used for each substream and that transmissions are organized into bursts of $L$ symbols. The power radiated by each transmitter is proportional to $1 / M$, so that the total radiated power is constant and independent of $M$ [2]. A straight forward way to implement coding for V-BLAST is to use the Horizontal Coding architecture shown in Fig.1. Each layer is encoded separately. The receiver can start V-BLAST detection and decoding from any layer, depending on the observed channel matrix. Then the receiver can perform interference cancellation using the decoding decisions from the previously decoded layers. One possible shortcoming of such approach is that the

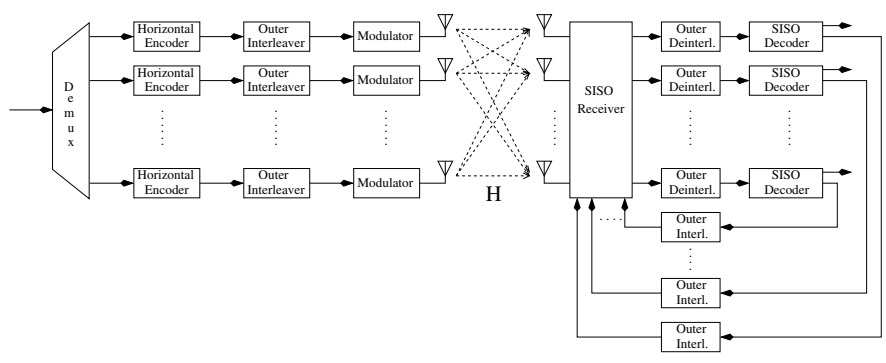

Fig. 1. Horizontal Coding Turbo-BLAST Scheme.

overall performance may be dominant by the weakest layer, particularly the first decoding layer because it has the lowest diversity in typical V-BLAST decoding. However, through iterative decoding and detection, the diversity order of each layer is ideally (assuming error free feedback) increased to the number of receiving antennas. The horizontal encoders can be quite generic, using block codes, convolutional codes, turbo codes, LDPC codes, or other error correcting codes.

The optimal receiver processing for a coded BLAST system requires a global ML solution, which jointly considers the detection (demodulation), deinterleaving and decoding of error correcting codes. However, due to the high complexity of 
such an approach many suboptimal techniques have been considered, including linear processing techniques such as Zero-Forcing (ZF) or Minimun Mean Square Error (MMSE) method, and non-linear methods such as Ordered Successive Interference Cancellation (OSIC). The performance of these suboptimal detection methods may be improved through iterative detection and decoding, which utilizes the decoding results for a second-round processing. It is assumed that the channel is varying slowly in time and that the communication is narrowband with the fading assumed to be frequency flat. At the receiver, the $\mathrm{N}$ receive antennas record an $\mathrm{N}$-dimensional complex vector superposition of the $M$ transmitted signals plus additive white Gaussian noise, as shown by:

$$
\mathbf{r}=\mathbf{H a}+\mathbf{v}
$$

where $\mathbf{H}^{N \times M}$ is the NxM matrix channel transfer function, and its element $h_{i j}$ is the complex transfer function from transmitter $j$ to receiver $i$, and $M \leq N$; the M-dimensional vector a contains the data substreams transmitted by antennas $1,2, \ldots, \mathbf{M}$, and $\mathbf{v}$ is the $N$ noise vector. The components of the noise vectors are uncorrelated zero-mean complex white Gaussian random variables with variance $\sigma^{2}$. To extract the desired signal, we perform Zero Forcing(or MMSE)-OSIC at the first step and interference cancellation and Maximal Ratio Combining (MRC) in a layer-by-layer fashion at the subsequent steps [4].

1) First Iteration: The full $\mathrm{ZF}(\mathrm{MMSE})-\mathrm{OSIC}$ V-BLAST detection algorithm can be described by a recursive procedure, including determination of the optimal ordering $\mathcal{S}_{\text {opt }}=\left\{k_{1}, k_{2}, \ldots, k_{M}\right\}$, as follows:

initialization:

$$
\begin{gathered}
i \leftarrow 1 \\
\mathbf{G}_{1}=\left\{\begin{array}{l}
\mathbf{H}^{+} \\
\left(\mathbf{H}^{\mathcal{H}} \mathbf{H}+\frac{\sigma^{2}}{E_{b} R_{c}} \mathbf{I}\right)^{-1} \mathbf{H}^{\mathcal{H}} \\
k_{1}=\arg \left\{\min _{j}\left\|\left(\mathbf{G}_{1}\right)_{j}\right\|^{2}\right\}
\end{array}\right.
\end{gathered}
$$

recursion:

$$
\begin{gathered}
\mathbf{w}_{k_{i}}=\left(\mathbf{G}_{i}\right)_{k_{i}} \\
y_{k_{i}}=\mathbf{w}_{k_{i}}^{T} \mathbf{r}_{i} \\
\hat{a}_{k_{i}}=Q\left(y_{k_{i}}\right) \\
\mathbf{r}_{i+1}=\mathbf{r}_{i}-\hat{a}_{k_{i}}(\mathbf{H})_{k_{i}} \\
\mathbf{G}_{i+1}=\left\{\begin{array}{c}
\widetilde{\mathbf{H}}_{k_{i}}^{+} \\
\left(\widetilde{\mathbf{H}}_{k_{i}}^{\mathcal{H}} \widetilde{\mathbf{H}}_{k_{i}}+\frac{\sigma^{2}}{E_{b} R_{c}} \mathbf{I}\right)^{-1} \widetilde{\mathbf{H}}_{k_{i}}^{\mathcal{H}}
\end{array}\right.
\end{gathered}
$$

$$
\begin{gathered}
k_{i+1}=\arg \left\{\min _{j \notin\left\{k_{1}, \ldots, k_{i}\right\}}\left\|\left(\mathbf{G}_{i+1}\right)_{j}\right\|^{2}\right\} \\
i \leftarrow i+1
\end{gathered}
$$

where $\left(\mathbf{G}_{i}\right)_{j}$ is the $j$ th row of $\mathbf{G}_{i}, \widetilde{\mathbf{H}}_{k_{i}}$ denotes the matrix obtained by zeroing columns $k_{1}, k_{2}, \ldots, k_{i}$, ${ }^{+}$denotes the Moore-Penrose Pseudoinverse, and Q(.) denotes the decision function. Thus, eq.(4) and (10) determine the elements of $\mathcal{S}_{\text {opt }}$, the optimal ordering; eq.(5)-(7) compute, respectively, the $\mathrm{ZF}(\mathrm{MMSE})$-nulling vector, the decision statistic and the estimated components of a; eq.(8) performs cancellation of the detected component from the received vector and, finally, eq.(9) computes the new pseudoinverse for the next iteration.

2) Subsequent Iterations: After the first iteration, interference cancellation is performed in a layer-by-layer fashion using the present soft decoding decisions for the already decoded sublayers and the previous iteration decisions for the others. The interference-free received vector pertaining to the $k$ th substream at the $m$ th iteration is

$$
\mathbf{r}_{k_{i}}^{(m)}=\mathbf{r}-\sum_{j=k_{1}}^{k_{i-1}} \mathbf{h}_{j} \hat{a}_{j}^{(m)}-\sum_{j=k_{i+1}}^{k_{M}} \mathbf{h}_{j} \hat{a}_{j}^{(m-1)}
$$

The decision statistic of the $k$ th substream obtained by performing the MRC on the interference-free received vector is given by

$$
y_{k_{i}}^{(m)}=\mathbf{h}_{k_{i}}^{\mathcal{H}} \mathbf{r}_{k_{i}}^{(m)}
$$

\section{A. MAP Decoding Algorithm}

We use parallel SISO decoders to provide the a priori probabilities of the transmitted substreams. In particular, SISO decoders use the bit-by-bit MAP decoding algorithm, well known as BCJR algorithm.

The a posteriori log-likelihood ratio $L\left(u_{k}\right)$ computed by the decoders can be splitted into three components, the extrinsic information $L_{e x}\left(u_{k}\right)$, the channel value and the a priori information $L_{a p}\left(u_{k}\right)$ :

$L\left(u_{k}\right) \triangleq \ln \left(\frac{P\left(u_{k}=+1 \mid \mathbf{y}\right)}{P\left(u_{k}=-1 \mid \mathbf{y}\right)}\right)=L_{e x}\left(u_{k}\right)+L_{c} y_{k}+L_{a p}\left(u_{k}\right)$

where $L_{c}=\frac{2 E_{b} R_{c}}{\sigma^{2}}$ is the reliability channel value.

$E_{b}$ denotes the received energy per information bit and $R_{c}$ is the used code rate.

\section{B. The Soft Decision Function $Q(\cdot)$}

The soft decision device $Q(\cdot)$ uses both the extrinsic information delivered by the SISO decoders at the previous iteration and the interference free decision statistic to provide a soft estimate of the received symbols:

$$
\hat{a}_{j}^{(m)} \triangleq Q\left(y_{j}^{(m)}\right)=\mathrm{E}\left\{a_{j} \mid y_{j}^{(m)},\left\{\mathrm{L}_{\mathrm{ex}}^{(\mathrm{m}-1)}\left(u_{k}\right)\right\}_{u_{k} \in a_{j}}\right\}
$$


Assuming that all bits $u_{i}$ forming the symbol $a_{j}$ are independent, we can write:

$$
\mathrm{P}^{(m)}\left(a_{j}=\alpha\right)=\prod_{u_{i} \in \alpha} \mathrm{P}^{(m)}\left(u_{i}\right)
$$

where $\alpha$ indicates a symbol belonging to the constellation set $\mathcal{A}$ of the modulators and $\mathrm{P}^{(m)}\left(u_{i}\right)$ can be formulated in terms of the bit extrinsic information as

$$
\mathrm{P}^{(m)}\left(u_{i}=+1\right)=\frac{\exp \left(\mathrm{L}_{\mathrm{ex}}^{(\mathrm{m}-1)}\left(u_{i}\right)\right)}{1+\exp \left(\mathrm{L}_{\mathrm{ex}}^{(\mathrm{m}-1)}\left(u_{i}\right)\right)}
$$

Finally, it can be shown that the eq.(15) can be rewritten as

$$
\hat{a}_{j}^{(m)}=\sum_{\alpha \in \mathcal{A}} \alpha \prod_{u_{i} \in \alpha} \mathrm{P}^{(m)}\left(u_{i}\right)
$$

The eq.(18)becomes very simple if a BPSK or a QPSK modulation is used:

C. $\Rightarrow B P S K$

$$
\begin{aligned}
& \hat{a}_{j}^{(m)}=\tanh \left[\frac{1}{2}\left(\frac{2 \sqrt{E_{b_{j}} R_{c}} y_{j}^{(m)}}{\sigma^{2}}+\mathrm{L}_{\mathrm{ex}}^{(\mathrm{m}-1)}\left(a_{j}\right)\right)\right] \\
& D . \Rightarrow Q P S K
\end{aligned}
$$

$$
\begin{aligned}
\hat{a}_{j}^{(m)} & =\tanh \left[\frac{1}{2}\left(\frac{2 \sqrt{E_{b_{j}} R_{c}} \Re\left(y_{j}^{(m)}\right)}{\sigma^{2}}+\mathrm{L}_{\mathrm{ex}}^{(\mathrm{m}-1)}\left(u_{1}\right)\right)\right]+ \\
& +j \tanh \left[\frac{1}{2}\left(\frac{2 \sqrt{E_{b_{j}} R_{c}} \Im\left(y_{j}^{(m)}\right)}{\sigma^{2}}+\mathrm{L}_{\mathrm{ex}}^{(\mathrm{m}-1)}\left(u_{2}\right)\right)\right]
\end{aligned}
$$

where $a_{j}=\left(u_{1}, u_{2}\right)$.

To reduce the computational complexity due to the nonlinear function $\tanh (\cdot)$, it can be approximated through 4-bits quantized values stored in a look-up table.

\section{A NEW SOFT CCK MOdem ARChIteCture}

The IEEE 802.11b standard adopts high data rate with bit rates up to $11 \mathrm{Mbps}$. For achieving data rate greater than 2 Mbps, the IEEE 802.11b standard specifies the Complementary Code Keying (CCK) modulation scheme. The IEEE 802.11 b complementary spreading codes have code length 8 and a chipping rate of $11 \mathrm{Mchip} / \mathrm{s}$. The 8 complex chips comprise a single symbol. By making the symbol rate 1.375 Msps, the 11 Mbps waveform ends up occupying the same approximate bandwidth as that for the 2 Mbps 802.11b QPSK waveform. The other IEEE $802.11 \mathrm{~b}$ requirements are:

- It operates in $2.4 \mathrm{GHz}-2.4835 \mathrm{GHz}$ frequency band

- Direct Sequence Spread Spectrum (DSSS)

- Three non-overlapping $22 \mathrm{MHz}$ channels

\section{A. Hard CCK Modulation}

The 8-bit CCK code words are derived from the following formula [5]:

$$
\begin{aligned}
& \mathbf{c}=\left\{e^{j\left(\varphi_{1}+\varphi_{2}+\varphi_{3}+\varphi_{4}\right)}, e^{j\left(\varphi_{1}+\varphi_{3}+\varphi_{4}\right)}, e^{j\left(\varphi_{1}+\varphi_{2}+\varphi_{4}\right)},-e^{j\left(\varphi_{1}+\varphi_{4}\right)},\right. \\
&\left.e^{j\left(\varphi_{1}+\varphi_{2}+\varphi_{3}\right)}, e^{j\left(\varphi_{1}+\varphi_{3}\right)},-e^{j\left(\varphi_{1}+\varphi_{2}\right)}, e^{j \varphi_{1}}\right\}
\end{aligned}
$$

This formula is used to generate the code sets for both 11 and 5.5 Mbps data rate. For the purpose of the discussion, just the $11 \mathrm{Mbps}$ mode will be described. Each data bit substream is partitioned into bytes as $\left(d_{7}, d_{6}, d_{5}, \ldots, d_{0}\right)$. The 8 bits are used to encode the phase parameters $\varphi_{1}-\varphi_{4}$ according to the scheme shown in Table I.

TABLE I

PHASE PARAMETER ENCODING SCHEME

\begin{tabular}{|c||c|}
\hline DIBIT & PHASE PARAMETER \\
\hline \hline$\left(d_{1}, d_{0}\right)$ & $\varphi_{1}$ \\
\hline$\left(d_{3}, d_{2}\right)$ & $\varphi_{2}$ \\
\hline$\left(d_{5}, d_{4}\right)$ & $\varphi_{3}$ \\
\hline$\left(d_{7}, d_{6}\right)$ & $\varphi_{4}$ \\
\hline
\end{tabular}

The encoding is based on a DQPSK modulation using Gray mapping rule.

\section{B. Hard CCK Demodulation}

For a complementary code which encodes $K M$-ary phases, ML decoding requires $M^{K-1}$ correlations. For the case where the number of phases $M$ is larger than 2, ML decoding quickly becomes too complex for practical implementation. Hence, less complex decoding techniques have been required. One way to decode the phases of a CCK code word is given by the following equations:

$$
\begin{aligned}
& \varphi_{2}=\arg \left\{-c_{1} c_{0}^{*}+c_{3} c_{2}^{*}-c_{5} c_{4}^{*}+c_{7} c_{6}^{*}\right\} \\
& \varphi_{3}=\arg \left\{c_{2} c_{0}^{*}-c_{3} c_{1}^{*}-c_{6} c_{4}^{*}+c_{7} c_{5}^{*}\right\} \\
& \varphi_{4}=\arg \left\{-c_{4} c_{0}^{*}-c_{5} c_{1}^{*}+c_{6} c_{2}^{*}+c_{7} c_{3}^{*}\right\} \\
& \varphi_{1}=\arg \left\{-c_{4} y_{4}^{*}+c_{2} y_{3}^{*}-c_{1} y_{2}^{*}+c_{0}\right\}
\end{aligned}
$$

where $y_{i}$ is the term within the $\arg \{\cdot\}$ expression of $\varphi_{i}$ in eq.(22).

The advantage of the above described decoding technique is that it provides automatic weighting of the subchannels; erroneous channels with low amplitudes will only give a minor contribution to the phase estimates. Even if equations (21) and (22) are simple to implement, they are not suitable for being used in an iterative structure because they don't make use of soft information delivered by the SISO decoders and don't provide the soft values needed by them. A new SOFT CCK Modem architecture has been derived and proposed in this paper to use the soft information provided by BLAST.

\section{Soft CCK Modulation}

The soft 8-bit CCK code words use the extrinsic information out coming from the decoders and are derived from the following formula:

$\tilde{\mathbf{c}}=\left\{\tilde{q}_{1} \tilde{q}_{2} \tilde{q}_{3} \tilde{q}_{4}, \tilde{q}_{1} \tilde{q}_{3} \tilde{q}_{4}, \tilde{q}_{1} \tilde{q}_{2} \tilde{q}_{4},-\tilde{q}_{1} \tilde{q}_{4}, \tilde{q}_{1} \tilde{q}_{2} \tilde{q}_{3}, \tilde{q}_{1} \tilde{q}_{3},-\tilde{q}_{1} \tilde{q}_{2}, \tilde{q}_{1}\right\}$ 
where the terms $\tilde{q}_{i}$ are the normalized soft DQPSK symbols reconstructed at the receiver through eq.(20).

\section{Soft CCK Demodulation}

The soft QPSK symbols are derived from the interference free normalized CCK code words $\tilde{\mathbf{c}}=\left\{\tilde{c}_{7}, \tilde{c}_{6}, \ldots, \tilde{c}_{0}\right\}$ by means the equations:

$$
\begin{aligned}
& q_{2}=-\tilde{c}_{1} \tilde{c}_{0}^{*}+\tilde{c}_{3} \tilde{c}_{2}^{*}-\tilde{c}_{5} \tilde{c}_{4}^{*}+\tilde{c}_{7} \tilde{c}_{6}^{*} \\
& q_{3}=\tilde{c}_{2} \tilde{c}_{0}^{*}-\tilde{c}_{3} \tilde{c}_{1}^{*}-\tilde{c}_{6} \tilde{c}_{4}^{*}+\tilde{c}_{7} \tilde{c}_{5}^{*} \\
& q_{4}=-\tilde{c}_{4} \tilde{c}_{0}^{*}-\tilde{c}_{5} \tilde{c}_{1}^{*}+\tilde{c}_{6} \tilde{c}_{2}^{*}+\tilde{c}_{7} \tilde{c}_{3}^{*} \\
& q_{1}=\frac{-\tilde{c}_{4} q_{4}^{*}+\tilde{c}_{2} q_{3}^{*}-\tilde{c}_{1} q_{2}^{*}}{4}+\tilde{c}_{0}
\end{aligned}
$$

\section{Performance Results in the IEEE $802.11 \mathrm{~b}$ CONTEXT}

This section presents the simulations results analyzing the behaviour and the performance of Horizontal coding TurboBLAST OSIC with ZF nulling strategies in the specific environment drawn by the standard IEEE $802.11 \mathrm{~b}$.

BER and FER performance versus $E_{b} / N_{0}$ have been derived numerically with different combination of the number of transmitter and receiver ${ }^{1}$, with different data rates and modulations, with variable packet length and number of Turbo-BLAST iterations as shown in Fig.2,3,4. The maximum performance of the T-BLAST receiver is achieved within about 4 or 5 iterations. Even in a slowly varying channel, the matrix channel transfer function $\mathbf{H}$ used in the receiver signal processing is considered constant and equal to the intermediate symbol time value into the packet. Transmission and receiving parameters are summarized in Table II.

TABLE II

SYSTEM PARAMETERS

\begin{tabular}{|l||c|}
\hline Carrier Frequency & $2.4 \mathrm{GHz}$ \\
\hline Chip Rate & $11 \mathrm{Mchip} / \mathrm{s}$ \\
\hline Mobile Velocity & $4 \div 5 \mathrm{Km} / \mathrm{h}$ \\
\hline Coding Mode & horizontal \\
\hline Code & Turbo $($ PCCC $)$ \\
\hline Const. Code Transfer Function & {$\left[1,\left(\frac{15}{13}\right)_{8}\right]$} \\
\hline Inner Interleaver & Block $($ standard UMTS $)$ \\
\hline Outer Interleaver & Helical \\
\hline Decoding Algorithm & Log $-M P$ \\
\hline
\end{tabular}

\section{ThroughPUt RESults IN THE IEEE 802.11B ENVIRONMENT}

Once the total transmitted power $P_{t}$ has been fixed, the SNR $E_{b} / N_{0}$ per information bit per receive antenna can be computed as

$$
\frac{E_{b}}{N_{0}}=\frac{P_{t} G_{T} G_{R}}{n R_{c} R_{b} N_{0} L}
$$

where $G_{T}$ and $G_{R}$ are respectively the transmit and receive antenna gain, $n$ is the number of transmitters, $R_{c}$ the code rate,

\footnotetext{
${ }^{1}$ for a given $E_{b} / N_{0}$, an increasing number of transmit antennas involves an increased total radiated power, while a greater number of receive antenna gets the total received energy per bit to increase, but with a constant value of radiated power.
}

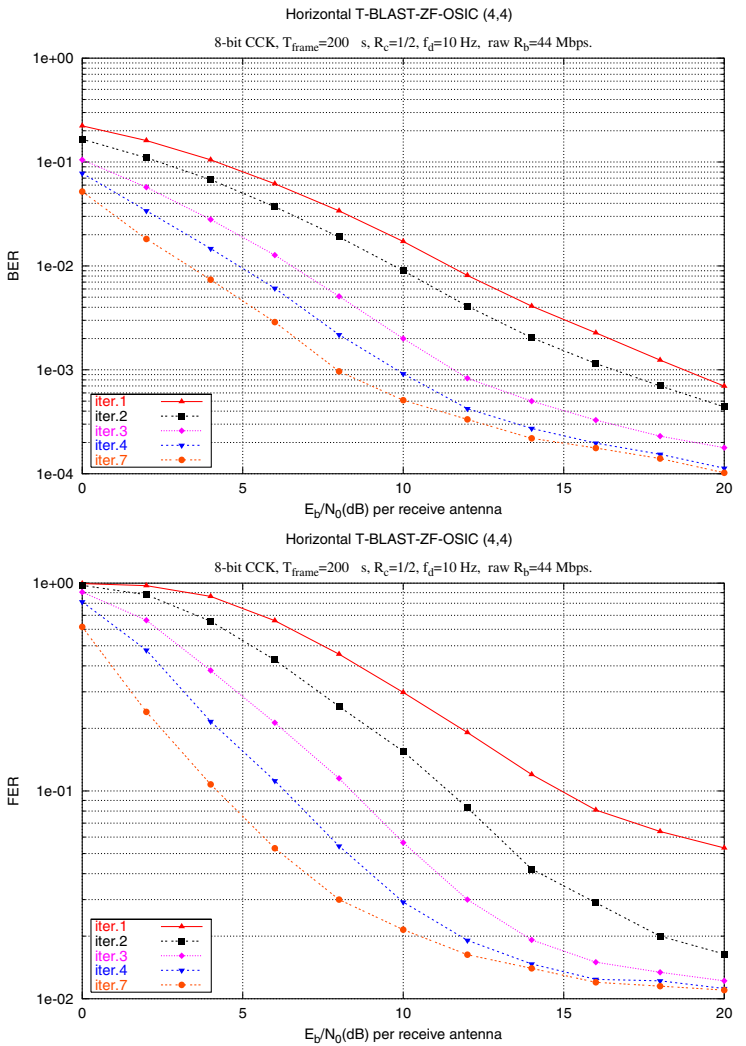

Fig. 2. Performance of Horizontal Turbo-BLAST-zf-osic with 8 bit-CCK modulation : the raw bit rate is $44 \mathrm{Mbps}$, while the payload bit rate is the $50 \%$ of the former.

$R_{b}$ the raw bit rate, $N_{0}$ the single side noise spectral density and $L$ the path-loss.

\section{A. Indoor Channel Models}

Based on experimental data, many approximated channel models have been developed to estimate indoor path loss, such as Logarithmic model, COST 231 model or the linear model. We consider the linear model path loss with an attenuation coefficient $a=0.47[\mathrm{~dB} / \mathrm{m}]$, typical in an office environment. The system parameters used in the following simulations are shown in Table III.

TABLE III

PARAMETERS FOR THROUghPUT CALCULATION FOR IEEE $802.11 \mathrm{~b}$

\begin{tabular}{|l|c|}
\hline Transmit Power & $100 \mathrm{~mW}$ \\
\hline Transmit Gain G & $0 \mathrm{~dB}$ \\
\hline Receive Gain $\mathbf{G}_{\mathbf{R}}$ & $0 \mathrm{~dB}$ \\
\hline Noise SpectraI Density $\mathbf{N}_{\mathbf{0}}$ & $-174 \mathrm{~dB}$ \\
\hline Path Loss Model & Linear $(a=0.47 \mathrm{~dB} / \mathrm{m})$ \\
\hline Frequency & $2.4 \mathrm{GHz}$ \\
\hline
\end{tabular}

Results shown in Fig. 6 suggest that for a fixed radiated power the throughput can be maximized both using a right modulation scheme and reducing the number of transmit antennas while the distance from receiver is increasing. 

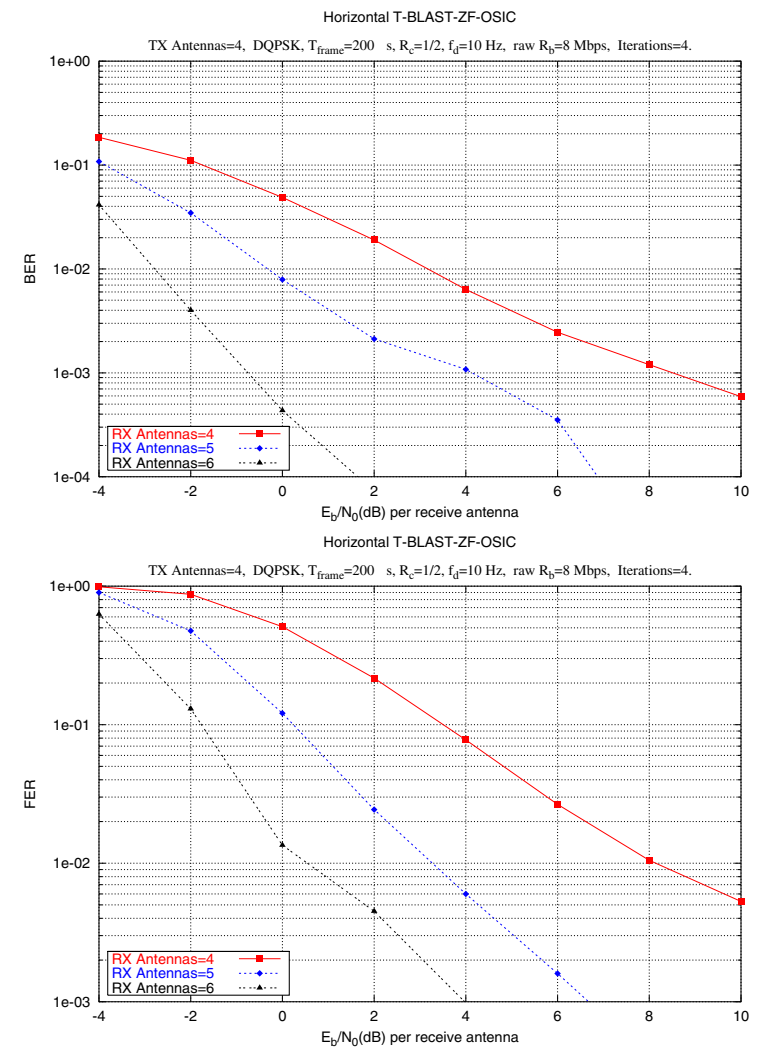

Fig. 3. Performance Gain of Horizontal Turbo-BLAST-zf-osic by increasing the number of Receive Antennas.

\section{CONCLUSIONS}

The main goal of this work is the combination of turbo principles and BLAST architecture in the IEEE $802.11 \mathrm{~b}$ environment. In the paper a modified 'SOFT' CCK modulation/demodulation has been derived, and this T-BLAST SOFT -CCK modem architecture improves the BER performance at each iteration in the IEEE $802.11 \mathrm{~b}$ standard environments. The evaluation results show that T-BLAST provides a reliable solution to high data rate transmission for wireless communications.

\section{REFERENCES}

[1] M. Sellathurai and S. Haykin, "Turbo-Blast for wireless communications:theory and experiments", IEEE Trans. on Signal Processing, V.50,n.10,October 2002.

[2] M. Sellathurai and S. Haykin, "Turbo-Blast high-speed wireless communications"', WCNC 2000, V.50,n.10,October 2002.

[3] A. van Zelst,R. van Nee and G.A. Awater, "Turbo-Blast and its performance", VTC 2001, V.50,n.10,October 2002.

[4] G. Golden,C.J. Foschini and R.A. Valenzuela, "Detection algorithm and initial laboratory VBLAST space time communication architecture", Electronics Letters, january 1999

[5] T.H. Kim,C.K. Kim and G.W. Chong, "A new architecture of CCK modem based on iterative differential modulation and phase detection", IEEE, 2001
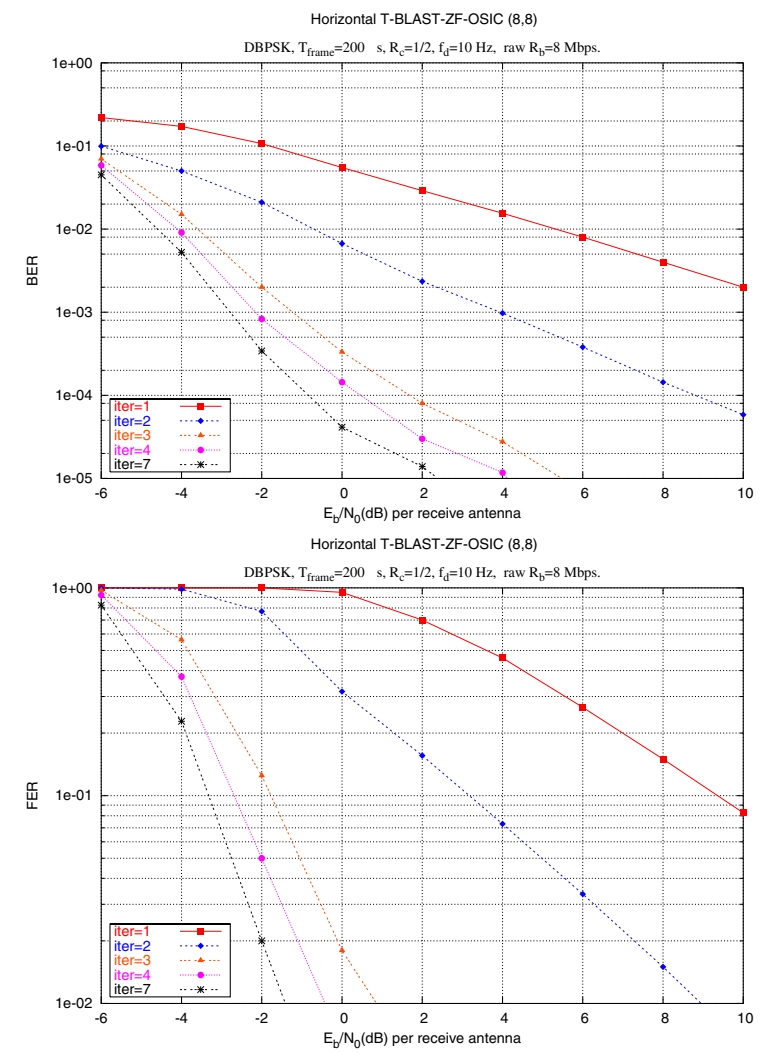

Fig. 4. Performance of Horizontal Turbo-BLAST-zf-osic with DBPSK modulation in a 8 Transmitter -8 Receiver system.

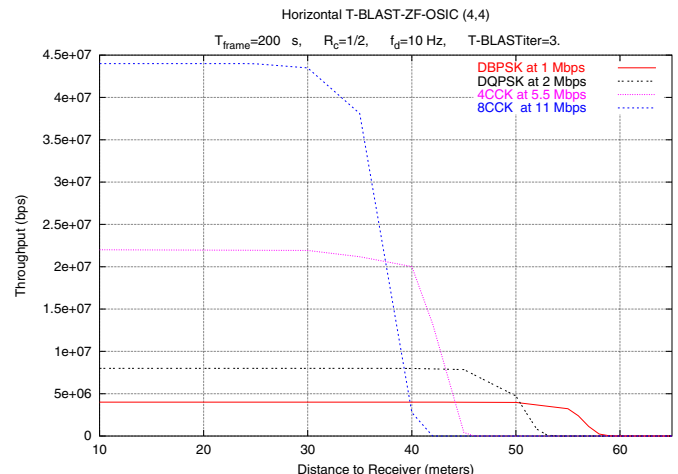

Fig. 5. Throughput versus Distance for different Modulation Schemes.

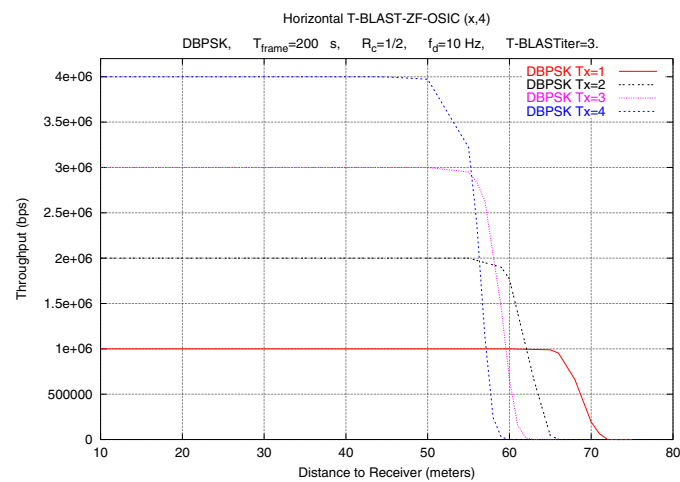

Fig. 6. Throughput versus Distance for a DBPSK Modulation varying the number of Transmit Antennas. 